\title{
BMJ Open Can trained volunteers improve the mealtime care of older hospital patients? An implementation study in one English hospital
}

\author{
Fiona F A Howson, ${ }^{1}$ Sian M Robinson, ${ }^{2}$ Sharon X Lin, ${ }^{3}$ Rosanna Orlando, ${ }^{3}$ \\ Cyrus Cooper, ${ }^{2}$ Avan A P Sayer, ${ }^{4,5}$ Helen C Roberts ${ }^{1,3,5}$
}

To cite: Howson FFA, Robinson SM, Lin SX, et al. Can trained volunteers improve the mealtime care of older hospital patients? An implementation study in one English hospital. BMJ Open 2018;8:e022285. doi:10.1136/ bmjopen-2018-022285

- Prepublication history for this paper is available online. To view these files, please visit the journal online (http://dx.doi. org/10.1136/bmjopen-2018022285).

Received 23 February 2018 Revised 4 May 2018 Accepted 28 June 2018
Check for updates

(C) Author(s) (or their employer(s)) 2018. Re-use permitted under CC BY-NC. No commercial re-use. See rights and permissions. Published by BMJ.

For numbered affiliations see end of article.

Correspondence to Professor Helen C Roberts; hcr@soton.ac.uk

\section{ABSTRACT}

Objective Multinational studies report undernutrition among 39\% older inpatients; importantly, malnutrition risk may further increase while in hospital. Contributory factors include insufficient mealtime assistance from time-pressured hospital staff. A pilot study showed trained volunteers could safely improve mealtime care. This study evaluates the wider implementation of a mealtime assistance programme.

Design Mixed methods prospective quasi-experimental study.

Setting Nine wards across Medicine for Older People (MOP), Acute Medical Unit, Orthopaedics and Adult Medicine departments in one English hospital.

Participants Patients, volunteers, ward staff.

Intervention Volunteers trained to help patients aged $\geq 70$ years at weekday lunchtime and evening meals.

Main outcome measures The number of volunteers recruited, trained and their activity was recorded. Barriers and enablers to the intervention were explored through interviews and focus groups with patients, ward staff and volunteers. The total cost of the programme was evaluated.

Results 65 volunteers (52 female) helped at 846 meals (median eight/volunteer, range 2-109). The mix of ages (17-77 years) and employment status enabled lunch and evening mealtimes to be covered. Feeding patients was the most common activity volunteers performed, comprising $56 \%$ of volunteer interactions on MOP and $34 \%-35 \%$ in other departments. Patients and nurses universally valued the volunteers, who were skilled at encouraging reluctant eaters. Training was seen as essential by volunteers, patients and staff. The volunteers released potential costs of clinical time equivalent to a saving of $£ 27.04 /$ patient/day of healthcare assistant time or $£ 45.04$ of newly qualified nurse time above their training costs during the study. Conclusions Patients in all departments had a high level of need for mealtime assistance. Trained volunteers were highly valued by patients and staff. The programme was cost-saving releasing valuable nursing time.

Trial registration number NCT02229019; Pre-results.

\section{INTRODUCTION}

Multinational studies report that 34\%-39\% of hospital inpatients aged over 65 years are at
Strengths and limitations of this study

- One of the largest studies of mealtime volunteers and the only one to formally evaluate the implementation of volunteers across four different clinical departments.

- Mixed methods design included cost analysis and evaluation of the context, barriers and enablers of the intervention from the perspective of patients, volunteers and staff.

- Limitations include that this was a single-site study, which did not include departments such as oncology or stroke units, where there may be a need for additional mealtime assistance.

risk of malnutrition. ${ }^{12}$ Further deterioration in dietary intake during a hospital admission may increase malnutrition risk, affecting up to $60 \%$ of older people. ${ }^{3}$ Malnutrition is expensive $^{4}$ as it is associated with poor healthcare outcomes including increased hospital admissions, longer length of stay and increased mortality. ${ }^{5}$ Many factors contribute to low food consumption while in hospital including the effects of acute illness, loss of appetite and side effects of medication. ${ }^{6} 7$ However, organisational factors associated with hospital admission are recognised to contribute including mealtime interruptions and lack of help at mealtimes, with time-pressured nursing staff facing competing clinical priorities. ${ }^{8}$ Coloured trays to indicate which patients need additional support at mealtimes are widely used but have had little formal evaluation. ${ }^{9}$ Studies of protected mealtimes where clinical activity is suspended have shown no increase in dietary intake for adult patients on medical and surgical wards, ${ }^{10}$ although sitting up for meals and timely mealtime assistance have been reported to be associated with greater intake. ${ }^{1112}$ 
Mealtime assistance includes cleaning patients' hands and tables before meals, helping open packets, cutting up food, encouraging reluctant eaters and feeding patients as well as promoting social interaction. Two recent systematic reviews based on small studies reported that trained volunteer mealtime assistants were safe and can improve the mealtime experience for patients although evidence for an increase in dietary intake was inconsistent. ${ }^{1314}$ Volunteers were perceived by patients and nurses to improve the quality of mealtime care ${ }^{15}$ but there was limited evidence of the cost-effectiveness of a volunteer programme. ${ }^{14}$ Importantly, those patients needing feeding assistance have been shown to be at the highest risk of poor energy and protein intakes. ${ }^{16}$ However, our current understanding of how to scale up small studies into hospital-wide clinical practice is limited, and no published literature to date has described this process with respect to volunteer mealtime assistance. In England, it is estimated that up to 3 million people are involved in voluntary work within health and social care, both in the voluntary sector and within public service. ${ }^{17}$ Many hospitals have volunteers who typically fulfil roles such as serving in cafes, greeting patients and visitors and running services such as radio and books distribution. Volunteers are perceived as an integral part of the care team in hospital and play an important role in improving patient experience in hospital. Volunteering is also recognised to be beneficial for the volunteers themselves; for example, lower mortality has been described among older volunteers. ${ }^{18}$ There is growing interest in the potential of trained volunteers supporting patients in hospitals in a variety of different ways. In the Hospital Elder Life Programme (HELP), volunteers were trained in six different areas including assistance with eating and drinking, with the aim to reduce incident delirium. ${ }^{19}$

This paper reports on the implementation of trained mealtime volunteers in nine wards in four different clinical departments in one hospital: medicine for older people (MOP) wards, acute medical unit (AMU), trauma and orthopaedics (T\&O) and adult medicine. The study aimed to determine the numbers of volunteers required to deliver mealtime assistance across these four departments and how best to recruit and retain volunteers; to describe and compare the activity and demographic profile of volunteers in the different clinical areas; to determine the barriers and enablers to the mealtime assistance programme from the perspectives of patients, nursing staff and the volunteers themselves and to assess the costs associated with the introduction of volunteer mealtime assistants across these hospital departments. This is the first study to report on large-scale implementation of a volunteer mealtime assistance programme and its cost implications.

\section{METHODS}

\section{Study design}

This was a mixed methods quasi-experimental prospective study with quantitative, qualitative and economic data analysis. As an evaluation of a complex intervention, this study follows the Medical Research Council (MRC) guidance for developing and evaluating complex interventions ${ }^{20}$ and builds on the earlier pilot study. ${ }^{21}$ As a complex intervention, the delivery of mealtime volunteer assistance was potentially influenced by the interactions with volunteers, the environment within the different departments, and the readiness and preferences of those patients receiving assistance. Thus, the MRC guidance on process evaluation of complex interventions was used to provide a framework for evaluating the implementation (how the intervention was delivered including its fidelity, quantity and reach of delivery in each department), mechanisms through which the intervention brought about change and context (external barriers and enablers to the implementation).$^{22}$ The results are reported in accordance with the Standards for Reporting Implementation Studies statement. ${ }^{23}$

\section{Patient involvement}

The volunteers' experiences from the pilot study ${ }^{20}$ helped shape the design of this study, alongside advice from the National Institute for Health Research Collaboration for Leadership in Applied Health Research and Care patient and public involvement team. Meetings with the volunteers were held at regular intervals to discuss study progress and later analysis and dissemination of results.

\section{Intervention}

Volunteers were recruited to work with patients aged $\geq 70$ years in four departments in one hospital: two male MOP wards, three AMU wards, two T\&O wards and two adult medical wards. This represented a range of case mix including patients living with frailty and dementia (MOP), acute illness (AMU), fractures (T\&O) and respiratory illness (adult medicine). Recruitment was conducted by the hospital's pre-existing voluntary services team, which comprises 2.5 full time equivalent members of staff and supported $>1000$ existing volunteers across the hospital. Recruitment strategies included advertising banners in main areas of the hospital, postcards by the cash registers in hospital cafes and restaurants, talks to local community groups and sixth form colleges (students aged 16-18) and hospital open days.

The volunteers attended a standardised half-day training session delivered by the research team on nutrition in older patients, safe feeding strategies, a practical session on feeding and assessment of competency prior to independent practice as previously described.$^{24}$ Following this competency assessment, the research team then met with each volunteer at the beginning of their next two mealtime shifts to ensure there were no concerns and that they were settled within the ward environment. The responsibility for identifying suitable patients for volunteer assistance was with the nurse in charge of each shift, and the volunteers handed over any relevant information to the nurse prior to their departure. Based on experience from the pilot study, we aimed to provide two volunteers to assist 
with weekday lunch or evening meals on each ward (10 volunteers/ward/week); the ward managers identified which mealtime was most useful. The exception was AMU where we aimed to provide assistance at both mealtimes. Volunteers were recruited to one clinical department at a time and matching of individual volunteers to wards was largely based on their preferred mealtime. In practice, college students were mainly available at evening mealtimes and older volunteers often preferred lunchtimes. The ward, day and mealtime of volunteering remained constant for each volunteer throughout the study and was decided at the volunteer's competency assessment. This enabled their integration into the ward team. Six monthly 'coffee and cake' meetings were held for the volunteers by the research team and voluntary services department, where the progress of implementation and any volunteer concerns were discussed. All volunteers were aware of how to contact the research team and voluntary services department by phone or email for any urgent concerns.

\section{Data collection}

Details of how the intervention was delivered, its fidelity, quantity and reach of delivery in differing departments were recorded in the following way. The numbers of volunteers at each stage of the recruitment process were recorded from initial contact, through training and competency, to mealtime assistance. At the end of each mealtime, volunteers recorded the number of patients they had helped and the manner of their assistance (defined for study purposes in ascending order as social interaction, encouragement, preparation, assisting food to mouth and finally feeding). Volunteers were trained in how to complete these activity forms and could record multiple options, although only the most in-depth assistance was noted by the research team. The proportion of mealtimes covered by volunteers across each department was recorded. Additionally, volunteers' demographic profile and reasons for volunteering were obtained through a brief self-completed anonymised questionnaire. Those who left the programme during the study were asked to complete a brief questionnaire to establish their reasons for leaving to inform future volunteer retention.

The mechanisms by which the intervention brought about change, the acceptability of the volunteer mealtime assistant programme in the different departments, and perceived barriers and enablers were assessed using qualitative methods. Semistructured interviews were conducted with a purposive sample of eight patients (men and women, aged $70-85$ years and over 85 years) and seven staff members representing different roles in all four departments who had frequent contact with the volunteers (four ward managers, a student nurse and two housekeepers). One focus group was held with nine volunteers working in a range of wards. The interviews and focus group were carried out by a single qualitative researcher, and all participants gave written informed consent. The context in which the volunteers worked in the four departments was further described by recruiting 50 patients in each department and collecting data on their demographic, clinical and nutritional characteristics (BMI, Malnutrition Universal Screening Tool (MUST) score, appetite). These participants also gave written informed consent.

The cost implications of the implementation were evaluated based on staff costs of training and administrative support. The duration and frequency of volunteer training sessions and competency assessments were recorded along with training staff seniority.

\section{Data analysis}

Quantitative data were double entered onto a database, cleaned and analysed in R using $\mathrm{R}$ Studio. ${ }^{25}$ Summary statistics-mean, SD; median, IQR and number, percentage (\%)-were used to describe the volunteer recruitment, activity, demographic profiles and retention as well as the patient characteristics. Differences between volunteers and patients in the four clinical departments were assessed using Kruskal-Wallis and $\mathrm{X}^{2}$ tests as appropriate. The interviews and focus group were audiotaped and transcribed verbatim. Analysis was conducted using the Framework Method, a method of analysis where qualitative data is summarised and analysed within a matrix of key themes. ${ }^{26}$ Two researchers independently coded the data and agreed on a thematic framework, after which one researcher indexed, charted, mapped and interpreted the data. Common themes and differences in the views and perceptions of patients, staff and volunteers in the four departments were identified.

The economic analysis was run in Microsoft Excel and assessed the economic implications of mealtime volunteers releasing staff time to undertake other clinical tasks. Inputs uncertainty and assumptions have been tested through a sensitivity analysis and outputs range estimated in volunteers releasing time for Band 3 and Band 5 nurses.

\section{RESULTS}

\section{Preparation for and process of implementation}

Agreement for large-scale implementation of the mealtime assistance programme was initially obtained from the hospital governing board, followed by dissemination by the research team to the different hospital departments via the hospital's Nursing and Midwifery Group. The research team then met with individual ward managers to discuss the programme, confirm the need for assistance and identify the most appropriate mealtime. Ward managers cascaded the information to their nursing teams, and the research team were able to provide further education to ward staff (if needed) at the time of volunteers' competency assessments. Implementation was facilitated by the successful pilot study, with preagreed processes in place for training and supervision of volunteers, as well as widespread knowledge within the hospital of the presence of a mealtime volunteer programme. 


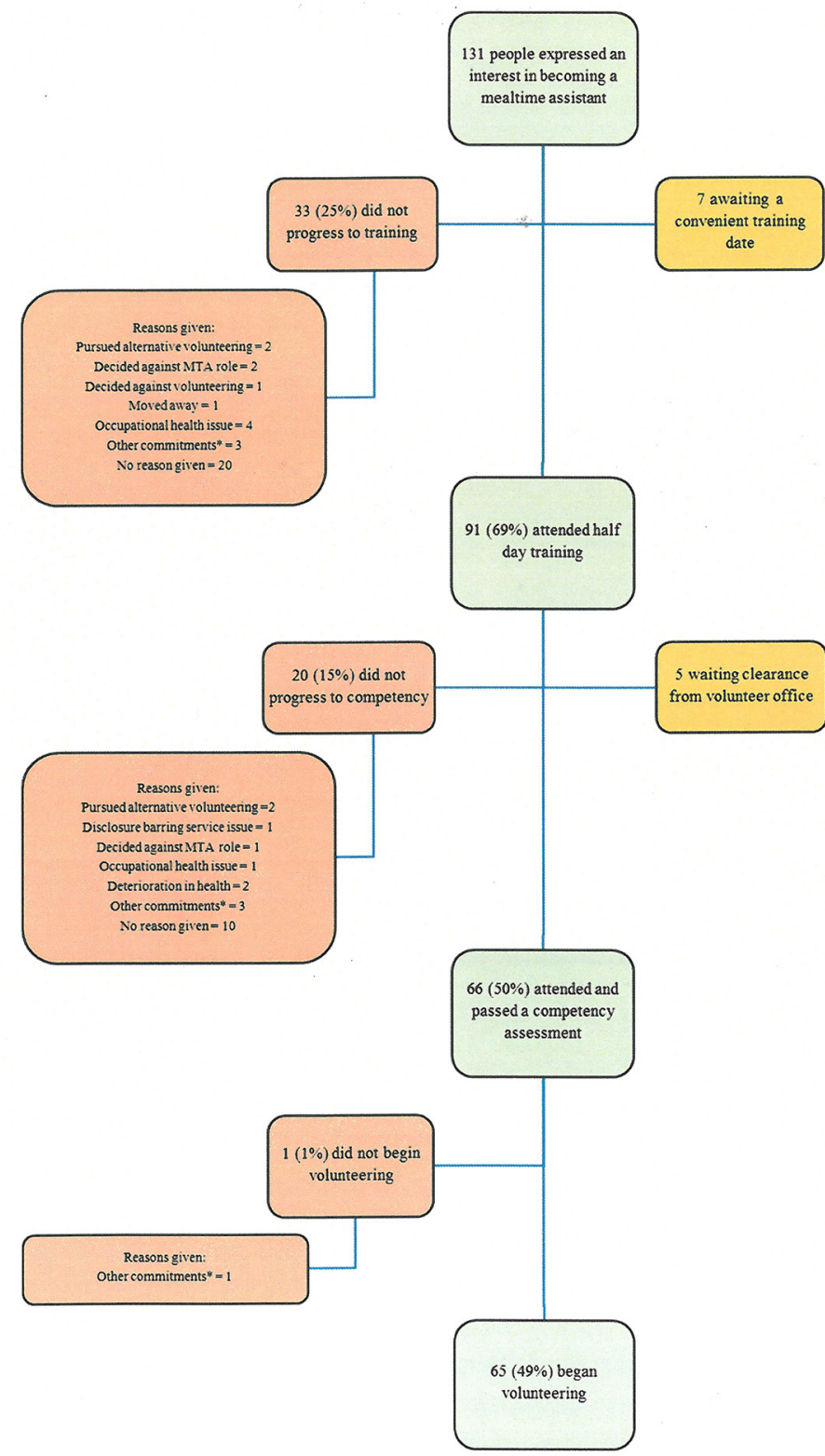

*Other commitments includes work study or family commitments

Figure 1 Volunteer recruitment and training.

\section{Delivery of the intervention}

During the 15-month study period, 131 people expressed an interest in providing mealtime assistance. Ninety-one people attended a training session and $65(70 \%)$ completed the subsequent competency assessment (figure 1). All 65 volunteers passed this competency assessment and went on to provide mealtime assistance. A further $12(13 \%)$ volunteers had a planned training session or competency assessment after data collection ceased and are not included in these results. However, $20(22 \%)$ of those trained did not attend the competency assessment, typically due to a change in the volunteer's health or other commitments. The median time between expressing an interest via the volunteer office and attending training was 31 days and between attending training and having a competency assessment was 26 days.

The demographic profile of the volunteers is shown in table 1 and was similar in each department. Fifty-two out of $65(80 \%)$ volunteers were female but the group included a range of different ages, ethnicity, working roles and previous experience. Students comprised the largest group of volunteers, often expressing an interest in a healthcare career and reflecting the success of the college talks, although advertising within the hospital was also successful. All volunteers were recruited by the hospital voluntary services team.

In total, 846 sessions were delivered by 65 volunteers during the study period (median eight sessions per 
Table 1 Volunteer characteristics by each department

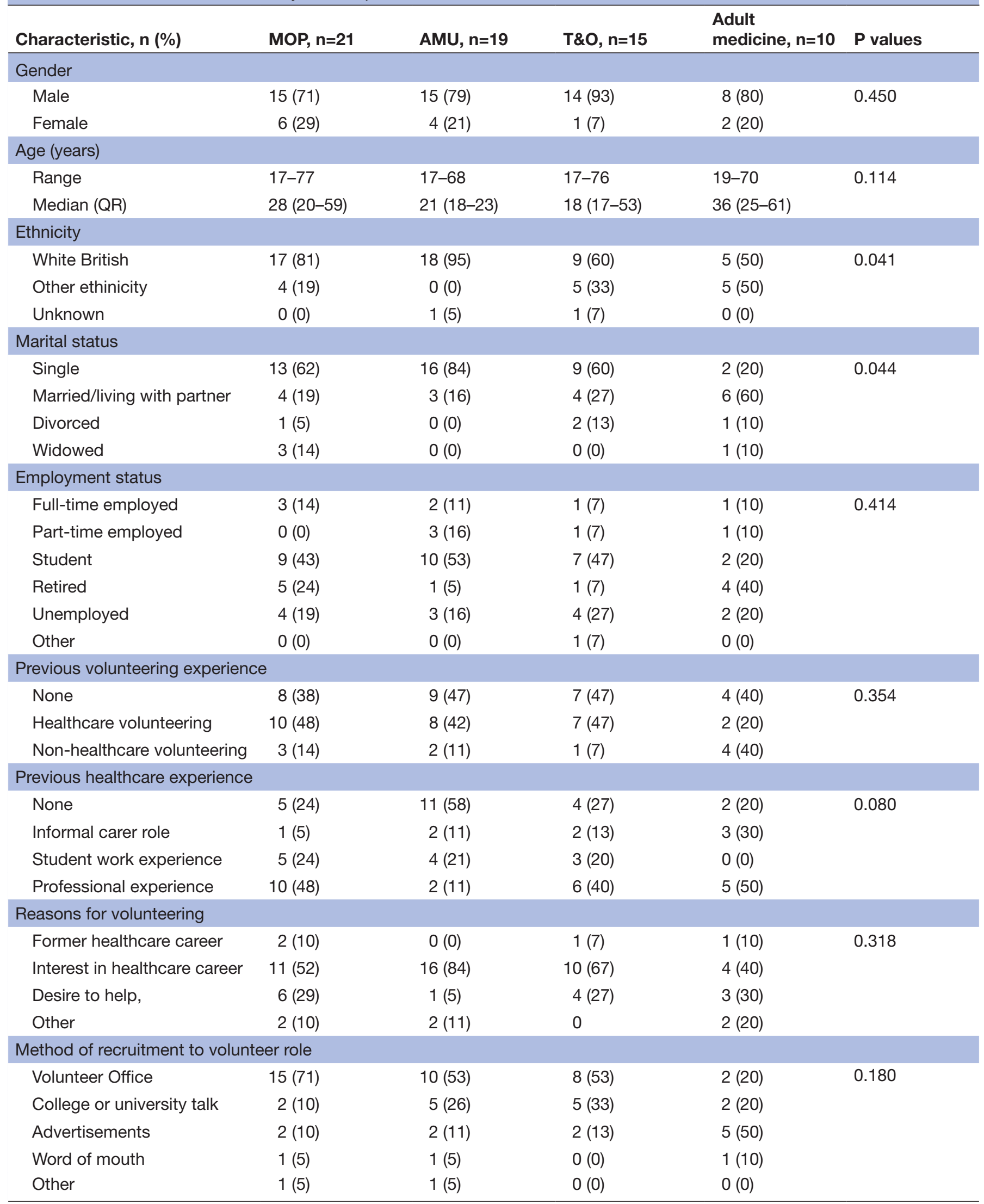

$P$ value calculated for age using Kruskal Wallis test; $\mathrm{p}$ values calculated for all other variables using $\mathrm{X}^{2}$ test.

AMU, acute medical unit; MOP, medicine for older people; T\&O, trauma and orthopaedics. 
Table 2 Volunteer activity recorded in each clinical department

\begin{tabular}{lcccc}
\hline & MOP & AMU & T\&O & Adult medicine P values \\
\hline Number of volunteers & 16 & 19 & 15 & 10 \\
Number of sessions & 313 & 167 & 121 & 54 \\
Duration of volunteering (weeks) & 68 & 54 & 35 & 19 \\
Activity, $n$ (\%) & & & \\
$\quad$ Social interaction & $12(2)$ & $53(8)$ & $57(14)$ & $18(13)$ \\
Encouragement & $104(19)$ & $68(11)$ & $30(8)$ & $25(18)$ \\
Preparation & $90(17)$ & $208(33)$ & $136(34)$ & $32(23)$ \\
Assisting food to mouth & $35(6)$ & $83(13)$ & $32(8)$ & $16(12)$ \\
Feeding & $305(56)$ & $225(35)$ & $395(100)$ & $138(100)$ \\
All & $546(100)$ & $637(100)$ & & $47(34)$ \\
\hline
\end{tabular}

$P$ value calculated using $X^{2}$ test.

$\mathrm{n}$, Number of patients assisted.

AMU, acute medical unit; MOP, medicine for older people; T\&O, trauma and orthopaedics.

volunteer, range 2-109). More sessions were delivered to MOP and AMU since the volunteers were recruited there first, and then in $\mathrm{T} \& \mathrm{O}$ and finally in adult medicine. Details of the type of mealtime assistance given to each patient by volunteers in each department was recorded by $60 / 65$ volunteers and relates to $655 / 846$ (77\%) sessions (table 2). In these 655 sessions, 1721 patients received mealtime assistance including 718 (42\%) whom the volunteers fed. Volunteers typically assisted 2-3 patients each session including feeding one person. There was a significant difference in the recorded assistance between the departments: the highest proportion of feeding assistance was in MOP (56\% compared with $34 \%-35 \%$ in other wards) while social interaction was more commonly recorded in $\mathrm{T} \& \mathrm{O}$ and adult medicine. There were also differences in recorded activity between volunteers. Social interaction and encouragement were recorded more often by volunteers aged $>25$ years $(10 \%$ and $15 \%$ activity, respectively) than those aged $<25$ years $(5 \%$ and $9 \%$, respectively). However, preparation of meals (eg, cutting up food and opening packets) was more frequently recorded by younger volunteers $(33 \%)$ than older ones $(25 \%)$. More experienced volunteers (defined as having delivered 12 or more sessions) also recorded assistance with preparation more frequently than the less experienced volunteers (29\% compared with 23\%). Finally, assisting food to mouth was more frequently reported by less experienced volunteers than more experienced volunteers ( $14 \%$ compared with $8 \%$ ). No adverse events were reported and there were no reports of patients refusing to be fed by volunteers.

During the study, the delivery of the intervention was adapted by one smaller ward for older patients and two wards with fewer older patients, each of which found that only one volunteer/mealtime was needed to provide sufficient mealtime assistance.

Thirty-four volunteers $(52 \%)$ continued volunteering at the end of the 15-month study period. The most common reasons for leaving, cited by 21 volunteers, were changes in work (8), study (4) or family (2) commitments and moving away (7). However, three volunteers stopped volunteering because ward refurbishment and consequent staff changes disrupted the relationships they had built with ward staff, and they did not wish to move to another ward team. There were no significant differences in reasons for leaving between the four departments. However, volunteers aged $<25$ years were significantly more likely than older volunteers to leave due to moving away (31\% compared with $13 \%$ ) and studying commitments (25\%: $0 \%)$ and less likely to leave due to work commitments $(6 \%: 47 \%)$ and changes to the ward environment $(0 \%: 20 \%)$.

\section{Context and barriers and enablers to the volunteer mealtime assistant programme}

There were important differences and similarities between the patients characterised in the four clinical departments (table 3). The MOP patients had a median age of 85 years compared with 74 years among the adult medicine patients. Patients characterised in these two departments were mostly male, while those in AMU and $\mathrm{T} \& \mathrm{O}$ had a higher proportion of women and of patients who lived alone. MOP patients had more comorbidities and lower Mini Mental State Examination scores, while the lowest Barthel scores were seen in T\&O. Importantly, the nutritional indices were similar across all four departments, with median BMI at or above the normal range and most patients having a low risk of malnutrition according to the MUST score. However, low median Simplified Nutritional Appetite Questionnaire (SNAQ) scores indicate significant anorexia among many patients in all departments.

Participants from all four departments identified common themes in interviews and the focus group. Ward staff reported that a lack of assistance at mealtimes was a factor in patients not eating enough prior to the introduction of the volunteers. Volunteers also recognised this as a problem and described the competing priorities that 
Table 3 Patient characteristics in each department

\begin{tabular}{|c|c|c|c|c|c|}
\hline Median (IQR) & MOP, $n=50$ & AMU, $n=50$ & T\&O, n=50 & Adult medicine, $n=51$ & P values \\
\hline Gender, n (\%) & & & & & $<0.001$ \\
\hline Female & $0(0)$ & $31(62)$ & $35(70)$ & $14(28)$ & \\
\hline \multicolumn{6}{|l|}{ Home situation, n (\%) } \\
\hline Living alone & $22(44)$ & $31(62)$ & $31(62)$ & $17(33)$ & \\
\hline Care home & $2(4)$ & 0 & $3(6)$ & 0 & 0.295 \\
\hline Personal care help & $16(32)$ & $16(32)$ & $7(14)$ & $5(10)$ & 0.004 \\
\hline Comorbidities & $7(5-8)$ & $6(4-8)$ & $4(3-6)$ & $5(4-7)$ & $<0.001$ \\
\hline Medications & $9(7-12)$ & $7(6-10)$ & $8(5-12)$ & $9(6-11)$ & 0.156 \\
\hline Body mass index $\uparrow$ & $24.3(22.0-27.1)$ & $26.9(23.0-30.3)$ & $25.6(21.9-28.4)$ & $25.4(22.5-29.1)$ & 0.131 \\
\hline MUST† low risk, n (\%) & $38(76)$ & $40(82)$ & $43(86)$ & $40(78)$ & 0.820 \\
\hline SNAQ & $15(12.5-16)$ & $13(12-15)$ & $14(12-15)$ & $14(912-15)$ & 0.227 \\
\hline
\end{tabular}

$P$ value calculated using Kruskal-Wallis test except for gender, home situation and MUST calculated using $X^{2}$ test.

${ }^{*} \mathrm{n}=49$ for $\mathrm{T} \& \mathrm{O}$.

$+\mathrm{n}=49$ for $\mathrm{AMU}$.

$\neq \mathrm{n}=49$ for MOP.

AMU, acute medical unit; MMSE, Mini Mental State Examination; MOP, medicine for older people; MUST, Malnutrition Universal Screening

Tool; SNAQ, Simplified Nutritional Appetite Questionnaire; T\&O, trauma and orthopaedics.

nurses had to face at mealtimes. Consistent with staff and volunteer views, most patients interviewed agreed that the nurses had too much to do to be able to provide effective help at mealtimes. Volunteers considered increasing patients' dietary intake as important. They recognised that this could be a significant challenge in some circumstances and had developed strategies to encourage patients to eat. All staff interviewed were positive about the volunteers and reported that they were often particularly good at encouraging reluctant eaters:

With a volunteer, the patients will eat a lot more, definitely, because they're getting that bit of conversation as well. Staff member 01

Volunteers felt that preparing patients for their meal was important, and both staff and volunteers identified the importance of the social aspects of the volunteer role to patients.

She'll help someone, but while she's talking to that patient, she's talking to the other three as well, so they're all sort of having a little chat while having their lunch. Staff member 02

It's not just the feeding, it's the social thing I think is as important as well. Volunteer 01

One member of staff reported that their ward had initially had some concerns about the volunteers but these had been quickly addressed once the volunteers started on the ward. No other staff members reported any concerns about the safety or appropriateness of volunteer feeding and many agreed that the routine of volunteers making contact with a staff member on arrival ensured that the assistance was appropriate. Staff wished volunteer numbers could be increased, but recognised that recruiting and maintaining a volunteer workforce was challenging. Volunteers valued the training they received, but reported that situations could arise that had not been discussed in the training session. One patient felt it was particularly important that volunteers were appropriately trained, managed and monitored.

\section{Cost analysis of the programme}

The cost analysis was based on the cost of staff resources required to implement the intervention. The implementation costs over the 15-month study period (August 2014-December 2015) included training sessions (13 in total), competency sessions (one per volunteer) and 1 hour of administrative support per volunteer (to encompass arranging training and support following attainment of competency). The total cost of implementation (recruiting and training the volunteers) was $£ 5681$, equivalent to $£ 87.40$ per volunteer. However, the volunteers released nursing time for clinical duties. The overall cost saving over and above the implementation costs (using NHS Agenda for Change Pay Scales, $2015)^{27}$ was $£ 17200$ saving if releasing Band 3 nurses and $£ 32400$ saving if releasing Band 5 nurses. This is equivalent to a potential cost saving per patient per day 
of $£ 27.04$ (Band 3) and $£ 45.04$ (Band 5) over the period of the study.

\section{DISCUSSION}

This is the first study to formally evaluate the implementation of a volunteer mealtime assistance programme at scale across a number of wards and different clinical departments. We used a range of methods to recruit volunteers and, in particular, talks to college students and advertising banners within the hospital were successful and supplemented the hospital voluntary service team's usual methods. Ninety-one out of 131 (70\%) people who expressed an interest attended a training session, of whom $70 \%$ subsequently worked as mealtime assistants. Training was essential and valued by patients, staff and volunteers. Sixty-five volunteers of different ages and experience worked across four clinical departments over a 15-month period: this mix of different ages was required to cover both lunch and evening mealtimes. The highest proportion of patients who were fed were in MOP but at least $34 \%$ volunteer activity in each department was feeding patients, which highlights a widespread need for mealtime assistance. The volunteer mealtime assistants were valued by staff and by patients in all four departments despite differences in patient ages. It was important to complete the training and clearances for volunteers swiftly in order to maintain their interest. There was a high turnover with almost half of both older and younger volunteers leaving by the end of study. This was mainly for personal reasons and the students finishing college, but a planned ward move and associated staff changes did contribute to three volunteers leaving. The recruitment, training, management and support of the volunteers took a significant amount of time and this was recognised by the ward staff. The 65 volunteers released valuable nursing time for clinical duties equivalent to $£ 17200$ of Band 3 healthcare assistant time and up to $£ 32400$ had the mealtime assistance time been given by newly qualified Band 5 nurses over the period of the study.

This study had a number of strengths. It is one of the largest studies of mealtime volunteers to date and the only one to formally evaluate the implementation of the volunteers in four different clinical departments. The mixed methods study design included a cost analysis and has evaluated the context, barriers and enablers to the intervention from the perspective of patients, staff and volunteers.

However, there were limitations to this study. It was carried out in one hospital in England with little ethnic diversity among the patients, although there was more diversity among the volunteers and staff. The focus on four departments meant that mealtime assistance for patients in other departments, such as those with cancer or stroke, was not evaluated. Volunteer activity was self-reported and not independently observed (although it was recorded for $77 \%$ of volunteer sessions), and it is therefore likely that recorded activity underestimates the scale of volunteer assistance. Finally, we did not measure the impact of volunteer mealtime assistance on dietary intake or nutritional status, as this has been reported in previous studies ${ }^{131416}$ and was not the focus of this implementation study.

Most previous studies of trained volunteer mealtime assistants for older people in hospital have been pilot or cross-sectional observational studies focused on measuring dietary intake, rather than implementation studies. Only two of these studies have included all patients on the study wards as in this study. ${ }^{1428}$ The benefit of collecting data at a ward level for an implementation study is that the practical implications for a hospital can be assessed. Several studies have reported recruiting students, ${ }^{29-31}$ and they were the sole workforce in two studies. ${ }^{32} 33$ Two studies have reported a similar training programme to the one developed for this study, consisting of a training session followed by observing the volunteers in practice. ${ }^{23} 34$ Other studies have similarly involved volunteers, feeding patients where needed. Only five published studies have formally assessed feedback from patients, volunteers or staff $^{1425283536}$ and in keeping with our study no paper reported any negative feedback. Two studies reported volunteer activity as the number of tasks and time spent with each patient and also found that volunteers spent more time with patients than nurses. ${ }^{23}{ }^{28}$ Only one study published in 2013 included an economic analysis and calculated a cost-savings in staff time for each volunteer activity, equivalent to US\$12-26 in staff costs for each encounter between volunteers and patients. ${ }^{23}$ In keeping with our study, two previous studies have reported a lack of adverse events with trained volunteer mealtime assistants working with older people. ${ }^{1423}$

The use of volunteers trained to provide mealtime assistance and its implementation has been assessed as part of a wider programme of volunteer activity in the HELP. $^{18}$ This intervention incorporated six different volunteer activities and was designed to reduce delirium rather than address a lack of mealtime assistance per se. When the implementation of HELP was evaluated across 17 hospital sites, $31 \%$ of hospitals adapted the volunteer feeding protocol, with some sites reducing the volunteer role to assistance with meal setup due to concerns about volunteers feeding patients. ${ }^{37}$ This is in direct contrast to our own study, where, apart from nurses on one ward voicing initial worries about volunteer feeding (that were rapidly allayed once the volunteers began), no concerns were raised and the implementation of feeding was not prevented. In line with our study, volunteer turnover in the HELP study necessitated constant recruitment and training of new volunteers.

This study has demonstrated that trained volunteers can deliver high-quality mealtime care, including safely feeding patients in a range of clinical departments where there was a high level of need. The volunteers released valuable nursing time for clinical tasks and the programme was very cost-effective. The trained mealtime volunteer programme has now been taken up by the hospital as part 
of its quality improvement framework. Other hospitals struggling to deliver mealtime care to older patients could develop similar trained mealtime volunteer programmes using the recruitment and training strategies outlined in this paper. It is important to note that the recruitment, management and retention of volunteer mealtime assistants was an active process needing dedicated personnel and resources from paid and voluntary staff with senior management support. The support of the hospital voluntary services team was essential. Retention was aided by timely post-recruitment checking of references, maintaining volunteers on one ward such that they became part of the ward team and ensuring volunteers were able to access help from the research team or voluntary services department when needed. Six monthly meetings with volunteers helped to maintain relationships.

While this study has demonstrated that volunteers can deliver quality mealtime care to older people in hospital at scale, there are other issues to be addressed to understand why patients eat so little when protected mealtimes and sufficient help is provided. In this and previous studies, it is clear that anorexia is a major problem among patients in each department and is rarely measured or addressed. Patients with confusion due to delirium and/or dementia have a high risk of poor nutrition and future research could address methods to improve this. Patient interviews suggest that a lack of physical activity in hospital also contributes to poor nutrition, with patients reporting that less should be eaten to balance the inactivity. ${ }^{15}$ Future research should evaluate whether volunteers can undertake other roles to support clinical staff including a focus on increasing the mobility of inpatients alongside mealtime assistance.

\author{
Author affiliations \\ ${ }^{1}$ Medicine for Older People, University Hospital Southampton NHS FT, Southampton \\ General Hospital, Southampton, UK \\ ${ }^{2}$ MRC Lifecourse Epidemiology Unit, University of Southampton, Southampton \\ General Hospital, Southampton, UK \\ ${ }^{3}$ NIHR CLAHRC Wessex, University of Southampton, Southampton General Hospital, \\ Southampton, UK \\ ${ }^{4}$ NIHR Newcastle Biomedical Research Centre, Newcastle University Newcastle \\ upon Tyne, UK \\ ${ }^{5}$ Academic Geriatric Medicine, University of Southampton, Southampton General \\ Hospital, Southampton, UK
}

Acknowledgements The contribution of the hospital voluntary services team, research nurses Debbie Speigal and Beth Giddins, the volunteers, Cynthia Russell public researcher and of the study participants is gratefully acknowledged.

Contributors HCR, FFAH, SMR, CC and AAS designed the study. FFAH led the data collection and volunteer training. SL and RO led on the statistical and economic data analysis, respectively. FFAH and HCR led on the qualitative data analysis. HCR wrote the first draft of the paper. All authors contributed to revised drafts and approved the final paper. HR is guarantor for the study. All authors, external and internal, had full access to all of the data (including statistical reports and tables) in the study and can take responsibility for the integrity of the data and the accuracy of the data analysis.

Funding This study was funded by the National Institute for Health Research (NIHR) Collaboration for Leadership in Applied Health Research and Care (CLAHRC) Wessex at the University of Southampton. This study was supported by the Faculty of Medicine and the Faculty of Health Sciences at the University of Southampton, and University Hospital Southampton NHS FT. HCR and SMR are supported by the
Southampton Biomedical Research Centre. AAS is supported by the NIHR Newcastle Biomedical research Centre. The views expressed are those of the authors and not necessarily those of the NHS, the NIHR or the Department of Health. The study funders and sponsor had no role in the design or conduct of this study

Competing interests None declared.

Patient consent Not required.

Ethics approval National Research Ethics Service Committee London, Chelsea (14/LO/1363).

Provenance and peer review Not commissioned; externally peer reviewed.

Data sharing statement The data are available on request by emailing the corresponding author.

Open access This is an open access article distributed in accordance with the Creative Commons Attribution Non Commercial (CC BY-NC 4.0) license, which permits others to distribute, remix, adapt, build upon this work non-commercially, and license their derivative works on different terms, provided the original work is properly cited, appropriate credit is given, any changes made indicated, and the use is non-commercial. See: http://creativecommons.org/licenses/by-nc/4.0/.

\section{REFERENCES}

1. Russell C, Elia M. Nutrition screening surveys in hospitals in England: BAPEN, 2014..

2. Kaiser MJ, Bauer JM, Rämsch C, et al. Frequency of malnutrition in older adults: a multinational perspective using the mini nutritional assessment. J Am Geriatr Soc 2010;58:1734-8.

3. Stratton RJ, Hackston A, Longmore D, et al. Malnutrition in hospital outpatients and inpatients: prevalence, concurrent validity and ease of use of the 'malnutrition universal screening tool' ('MUST') for adults. Br J Nutr 2004;92:799-808.

4. Elia M. The cost of malnutrition in Engand and potential cost savings from nutritional interventions: BAPEN, Redditch:, 2015.

5. Guest JF, Panca M, Baeyens JP, et al. Health economic impact of managing patients following a community-based diagnosis of malnutrition in the UK. Clin Nutr 2011;30:422-9.

6. Patel MD, Martin FC. Why don't elderly hospital inpatients eat adequately? J Nutr Health Aging 2008;12:227-31.

7. Pilgrim AL, Baylis D, Jameson KA, et al. Measuring appetite with the simplified nutritional appetite questionnaire identifies hospitalised older people at risk of worse health outcomes. J Nutr Health Aging 2016;20:3-7.

8. Walton $\mathrm{K}$, Williams $\mathrm{P}$, Tapsell $\mathrm{L}$, et al. Observations of mealtimes in hospital aged care rehabilitation wards. Appetite 2013;67:16-21.

9. Roberts HC. Changing the food environment: the effect of trained volunteers on mealtime care for older people in hospital. Proc Nutr Soc 2018;77:95-9.

10. Hickson M, Connolly A, Whelan K. Impact of protected mealtimes on ward mealtime environment, patient experience and nutrient intake in hospitalised patients. J Hum Nutr Diet 2011;24:370-4.

11. Palmer M, Huxtable S. Aspects of protected mealtimes are associated with improved mealtime energy and protein intakes in hospitalized adult patients on medical and surgical wards over 2 years. Eur J Clin Nutr 2015;69:961-5.

12. Young A, Allia A, Jolliffe L, et al. Assisted or protected mealtimes? Exploring the impact of hospital mealtime practices on meal intake. $J$ Adv Nurs 2016;72:1616-25.

13. Tassone EC, Tovey JA, Paciepnik JE, et al. Should we implement mealtime assistance in the hospital setting? A systematic literature review with meta-analyses. J Clin Nurs 2015;24(19-20):2710-21.

14. Howson FFA, Sayer AA, Roberts HC. The impact of trainedvolunteer mealtime assistants on dietary intake and satisfaction with mealtime care in adult hospital inpatients: a systematic review. J Nutr Health Aging 2017;21:1038-49.

15. Robison J, Pilgrim AL, Rood G, et al. Can trained volunteers make a difference at mealtimes for older people in hospital? A qualitative study of the views and experience of nurses, patients, relatives and volunteers in the Southampton Mealtime Assistance Study. Int $J$ Older People Nurs 2015;10:136-45.

16. Roberts HC, Pilgrim AL, Jameson KA, et al. The impact of trained volunteer mealtime assistants on the dietary intake of older female in-patients: The Southampton mealtime assistance study. J Nutr Health Aging 2017;21:320-8.

17. Volunteering in health and care: Securing a sustainable future. 2018 www.kingsfund.org.uk/publications/volunteering-health-and-care.

18. Okun MA, Yeung EW, Brown S. Volunteering by older adults and risk of mortality: a meta-analysis. Psychol Aging 2013;28:564-77. 
19. Inouye SK, Bogardus ST, Baker DI, et al. The Hospital Elder Life Program: a model of care to prevent cognitive and functional decline in older hospitalized patients. Hospital Elder Life Program. J Am Geriatr Soc 2000;48:1697-706.

20. Craig P, Dieppe P, Macintyre S, et al. Developing and evaluating complex interventions: the new Medical Research Council guidance. BMJ 2008;337:a1655

21. Roberts HC, Pilgrim AL, Elia M, et al. Southampton Mealtime Assistance Study: design and methods. BMC Geriatr 2013;13:5.

22. Moore GF, Audrey S, Barker M, et al. Process evaluation of complex interventions: Medical Research Council guidance. BMJ 2015;350:h1258.

23. Pinnock H, Barwick M, Carpenter CR, et al. Standards for Reporting Implementation Studies (StaRI): explanation and elaboration document. BMJ Open 2017; 7:e013318.

24. Roberts HC, De Wet S, Porter K, et al. The feasibility and acceptability of training volunteer mealtime assistants to help older acute hospital inpatients: the Southampton Mealtime Assistance Study. J Clin Nurs 2014;23:3240-9.

25. Team RDC. R: A language and environment for statistical computing. Vienna, Austria: R Foundation for Statistical Computing, 2016.

26. Gale NK, Heath G, Cameron E, et al. Using the framework method for the analysis of qualitative data in multi-disciplinary health research. BMC Med Res Methodol 2013;13:117.

27. Curtis L, Burns A. Unit costs of health and social care 2015. Personal Social Services Research Unit, University of Kent, Canterbury 2015.

28. Huxtable S, Palmer M. The efficacy of protected mealtimes in reducing mealtime interruptions and improving mealtime assistance in adult inpatients in an Australian hospital. Eur $\mathrm{J}$ Clin Nutr 2013;67:904-10.

29. Buys DR, Flood KL, Real K, et al. Mealtime assistance for hospitalized older adults: a report on the SPOONS volunteer program. J Gerontol Nurs 2013;39:18-22.

30. Sneddon J. Using mealtime volunteers to support patients. Nurs Times 2011;107:21-3.

31. Robinson S, Clump D, Weitzel T, et al. The Memorial Meal Mates: a program to improve nutrition in hospitalized older adults. Geriatr Nurs 2002;23:332-5.

32. Wright L, Cotter D, Hickson M. The effectiveness of targeted feeding assistance to improve the nutritional intake of elderly dysphagic patients in hospital. J Hum Nutr Diet 2008;21:555-62.

33. Gilbert J, Appleton A, Jerrim J, et al. Assisted feeding for elderly inpatients. Clin Med 2013;13:324.

34. Manning F, Harris K, Duncan R, et al. Additional feeding assistance improves the energy and protein intakes of hospitalised elderly patients. A health services evaluation. Appetite 2012;59:471-7.

35. Walton $\mathrm{K}$, Williams $\mathrm{P}, \mathrm{Bracks} \mathrm{J}$, et al. A volunteer feeding assistance program can improve dietary intakes of elderly patients--a pilot study. Appetite 2008;51:244-8.

36. Huang CS, Dutkowski K, Fuller A, et al. Evaluation of a pilot volunteer feeding assistance program: influences on the dietary intakes of elderly hospitalised patients and lessons learnt. $J$ Nutr Health Aging 2015;19:206-10.

37. Inouye SK, Baker DI, Fugal P, et al. Dissemination of the hospital elder life program: implementation, adaptation, and successes. J Am Geriatr Soc 2006;54:1492-9. 